\title{
Travailler langue et culture en jouant avec les lieux, le savoir et la narration
}

\section{Learning about culture and language through the exploration of places, knowledge and storytelling}

\author{
Christine Evain \\ Université Rennes 2 \\ christine.evain@univ-rennes2.fr
}

\begin{abstract}
The storytelling activity coupled with that of playing and sharing a 'story' is part of the traditional repertoire of language didactics. In the context of a language project, a group of foreign Master's students at the Ecole Centrale de Nantes were asked to use two tools: the Baludik (a gamified circuit editor) and eZoomBook (an enriched book editor). Our article reports back on the methodology used to offer students creative activities based on the Baludik and eZoomBook editors, and analyses the students' results and feedback. Our goal is to suggest possible pedagogical uses of these circuits and book editors, applicable to the learning of different foreign languages.
\end{abstract}

Keywords: Baludik, eZoomBook, multi-layered narration, geocatching, scénographie

\section{INTRODUCTION}

L'activité de raconter couplée à celle de jouer et de partager autour d'une «histoire» fait partie du répertoire traditionnel de la didactique des langues. De nombreux chercheurs ont mis en lumière les bénéfices de la lecture non seulement pour approfondir un regard sur le monde mais également pour travailler l'expression orale et écrite.

Dans le cadre d'un projet linguistique, les étudiants étrangers de Master à l'Ecole Centrale de Nantes ont été invités à utiliser deux outils que sont les éditeurs Baludik et eZoomBook. Le premier permet de créer des parcours ludiques 
en lien avec une thématique donnée, et le second est un outil de construction de livre digital comportant plusieurs niveaux de lecture, avec des allers et retours possibles entre les différents niveaux. Nous souhaitons rendre compte non seulement de la méthodologie utilisée pour proposer aux étudiants un travail s'appuyant sur les éditeurs Baludik et eZoomBook mais également du retour des étudiants, mesuré par une enquête effectuée auprès d'une centaine d'entre eux. Notre objectif est de proposer des pistes de travail pour une utilisation pédagogique de ces deux éditeurs de parcours et de livre numérique multimodal, applicable à l'apprentissage de différentes langues étrangères.

Nous nous appuierons, d'une part, sur la recherche concernant les avantages des outils numériques et, d'autre part, sur l'approche recherche-action permettant d'effectuer un retour d'expérience et de proposer des améliorations pour les futures éditions de notre projet.

Constats préliminaires :

Le cours de Master "Cultural and Business Environment» (CBE) qui est le cadre de notre expérience est à la fois un cours de langue pour un groupe $\mathrm{d}$ 'apprenants très hétérogènes (allant du $\mathrm{C} 1$ aux étudiants bilingues) et un cours sur l'environnement culturel et commercial dans les différents pays de langue anglaise. Ces pays comprennent les pays anglo-saxons et les anciennes colonies britanniques dans lesquelles la langue anglaises est une des langues nationales.

Si l'attente des étudiants diffère sur le plan linguistique (en fonction des profils d'apprenants), elle converge sur le plan du contenu professionnel et du maniement des outils numériques. En effet, les étudiants souhaitent tous que le cours de CBE soit en lien avec l'entreprise : bien que leur attente soit essentiellement communicationnelle, ils expriment une certaine fascination pour les start-ups et développements technologiques dans le domaine du numérique. Pour ce qui est de la lecture, ils se situent dans la tendance actuelle, décrite par Bon (2011) qui propose de « dépasser le manque d'épaisseur » du livre en faisant «surgir des textes » (Bon, 2011) et des enrichissements reliés par hyperliens au texte initial ${ }^{1}$.

L'objectif de notre projet est double : il s'agit premièrement de progresser en langues grâce à des activités de lecture, d'échanges oraux et d'écriture. La progression des étudiants n'a pas été mesurée autrement que par des entretiens qualitatifs et un questionnaire en fin de parcours ${ }^{2}$. Ainsi nous nous sommes intéressés principalement à la motivation et la perception du projet par les étudiants.

${ }^{1}$ « Comment dépasser le manque d'épaisseur que procure le livre ? On commence à s'y risquer. Utiliser une navigation graphique au lieu d'une table des matières textuelles, utiliser les sous-couches de l'epub pour faire surgir des textes qui s'ouvrent depuis un point précis du texte initial... Le verrou est plutôt à l'intérieur de nous-mêmes » Bon (2011). Bon souligne bien cet aspect, en interrogeant non seulement le texte littéraire, mais aussi la place du lecteur, le sujet lecteur.

${ }^{2}$ Pour la progression en langues, aucune méthodologie d'évaluation scientifique ne pouvait être mise en place à cause de la combinaison de trois facteurs : le temps imparti était trop court, le groupe 
Quant à notre deuxième objectif, il porte sur les genres du discours, notre consigne aux étudiants étant de créer un genre, hybride, improbable : une narration eZoomBook couplée à un circuit Baludik. Concernant cette deuxième composante $\mathrm{du}$ projet, un retour quantitatif et qualitatif nous a permis d'analyser l'intérêt des étudiants et proposer des améliorations pour le renouvellement du projet.

\section{PRÉSENTATION DES OUTILS}

\section{Baludik :}

Baludik est une application de " géocaching » (une chasse au trésor géolocalisée) qui a été créée par une start-up nantaise, Baludik, dirigée par Jérémie Simon. L'application permet de créer et d'administrer des circuits avec des étapes, des questions et des énigmes à résoudre. Pour les utilisateurs ou « joueurs », les circuits se présentent sous la forme d'un jeu de piste digital : lorsqu'un joueur atteint un emplacement spécifique, un système GPS confirme son emplacement et lui pose des questions. S'il trouve la réponse, le téléphone émet des bips sonores : l'étape suivante est débloquée, et les joueurs reçoivent leur prochain indice.

L'interface de création de jeu de piste sur la plateforme Baludik est très simple d'utilisation. En effet le créateur du circuit est guidé pas à pas pour insérer les questions et les indices de son choix. Ces questions peuvent être des questions culturelles ou tout type de question que le créateur du circuit souhaite inclure. Pour notre « Baludik Challenge », nous avons mis l'accent sur les questions culturelles. Une fois prêt, le circuit est publié et mis à la disposition de tous les joueurs de la plate-forme. Pour accéder au contenu d'un circuit donné, les joueurs doivent se trouver à l'endroit précis où commence le circuit.

Lors du lancement de notre projet, nous avons testé des jeux existants sur la plateforme Baludik. Puis, nous avons accueilli Jérémie Simon qui a proposé une conférence-formation sur l'outil éditeur. Ainsi, les étudiants ont pu découvrir l'ensemble des fonctionnalités de l'application (par exemple comment insérer flash codes, contenu et indices multimédia, et comment programmer les différentes étapes du circuit jusqu'à la validation de la mission).

\section{eZoomBook :}

Un eZoomBook est un livre électronique à plusieurs niveaux incorporant différentes couches de contenu accessibles par des onglets dans un menu. Les eZoomBooks incluent une fonction de zoom avant et arrière permettant aux lecteurs de naviguer entre les différentes couches. L'outil eZoomBook a déjà été utilisé pour un certain nombre de projets pédagogiques dans l'enseignement supérieur, primaire

trop hétérogène, et l'activité linguistique trop diffuse pour pouvoir tirer véritablement des conclusions sur le plan des progrès en langues. 
et secondaire (Evain, De Marco, Carolan, 2013 ; Evain, De Marco, 2014, 2015, 2016 ; Bellay, Evain, De Marco, 2017 ; Iwaszko, Lojkine, Evain, De Marco, 2018). Comme l'indiquent ces articles, le logiciel a été créé par des étudiants et des enseignants à ECN et a été utilisé dans de nombreux types d'applications en lien avec la littérature et la science. L'éditeur eZoomBook permet à n'importe qui de créer un eZoomBook et de personnaliser le menu. Ainsi, les onglets peuvent inclure : une œuvre originale, des résumés, du vocabulaire (mots et expressions), des traductions, des jeux, des images, des liens vers des ressources en ligne (texte, image, vidéo, audio). L'utilisation de l'éditeur eZoomBook en cours de langues a pour principal avantage de promouvoir la lecture et l'écriture, de faciliter la découverte d'un patrimoine par l'exploitation des ressources multimédia (Louichon, $2015)^{3}$. eZoomBook permet également de développer du matériel pédagogique à usages multiples (cibles, objectifs d'apprentissage et contextes différents). Enfin, il encourage les élèves ou étudiants à «apprendre à apprendre » en leur donnant davantage de contrôle sur le processus d'apprentissage et en faisant apparaître le lien entre l'apprentissage ou la maîtrise d'une langue et la lecture.

\section{MÉTHODOLOGIE DE RECHERCHE}

Notre projet se situe dans une perspective recherche-action (R-A) ainsi définie par Narcy-Combes (1998, p. 230) : « une recherche liée à la réalisation pratique de résolution de problèmes de terrain où la réflexion a une dimension aussi bien pratique que critique $»$. Les étapes d'une telle recherche sont : premièrement, la mise en place d'un projet, deuxièmement, son analyse (observation, collecte de données, interprétation) et, enfin, une rétroaction (proposition de pistes d'amélioration). Ces étapes nécessitent une implication du terrain, un travail en équipe ainsi que l'humilité du chercheur qui ne se situe pas dans une posture hiérarchique mais collaborative. C'est peut-être « parce que cette conception [de la recherche comme recherche-action] inclut le principe de comptes à rendre (accountability), [qu'] elle semble particulièrement responsabilisante »(Narcy-Combes, 2001, p. 123). Ainsi nous avons procédé en trois temps :

- lancement et déroulement du projet, en nous appuyant sur les consignes énoncées ci-dessous ;

- collecte de données :

- travaux réalisés par les étudiants,

\footnotetext{
${ }^{3}$ Selon Louichon, les possibilités offertes par les ressources numériques permettent justement de mettre à disposition de manière intéressante les textes du patrimoine, dont la lecture et l'enseignement doivent être guidés et accompagnés (Louichon, 2015). La multimodalité donne par exemple la possibilité d'intégrer les OSS (Objets Sémiotiques Secondaires), créant ainsi une dialectique féconde entre l'œuvre patrimoniale et sa réception contemporaine.
} 
- observations des enseignants,

- feedback qualitatif et quantitatif (discussions avec les étudiants ; questionnaire, etc.) ;

- recommandations pour le renouvellement du projet.

Cette démarche en trois temps par laquelle nous situons dans une approche R-A, revient à adopter une posture pragmatique : l'analyse des forces et des faiblesses du projet débouche sur des propositions d'améliorations pour les éditions futures de ce projet.

Instructions aux élèves et objectifs des enseignants :

Dans le cadre de ce projet, l'équipe de quatre enseignants avaient pour objectif de donner aux étudiants l'occasion de travailler ensemble et de développer diverses compétences de collaboration : gestion, leadership, constitution d'équipes, créativité, observation et résolution de problèmes, positionnement de marque. Nous souhaitions que les étudiants expérimentent les éditeurs eZoomBook et Baludik, dans le contexte du projet, puis en évaluent la pertinence, l'efficacité et l'impact motivationnel.

Les étudiants devaient s'organiser pour que deux groupes fonctionnent en tandem afin de produire à la fois un eZoomBook et un circuit Baludik ${ }^{4}$. Pour commencer, il leur a fallu s'approprier l'outil puis travailler sur leurs propres contenus, en lisant, recherchant, résumant, présentant, expliquant, traduisant, des informations de leur choix et, pour les équipes "eZoomBook», en injectant une composante fictionnelle. À la fin du semestre, pendant l'événement appelé « défi Baludik », les étudiants devaient tester leurs circuits respectifs, selon un ensemble de règles définies par un groupe d'étudiants appelé « les organisateurs ». L'évaluation reposait sur l'évaluation par les pairs et l'évaluation par les enseignants d'une présentation des travaux des groupes en fin de semestre. Pour la journée du défi Baludik, la liste des exigences relatives au circuit comprenait un certain nombre d'éléments, par exemple :

- le circuit devait durer environ $3 / 4$ d'heure et un minimum de 3 arrêts (avec les coordonnées GPS), à distance de marche les uns des autres ;

- les questions sur le circuit devaient être liées à un thème donné et à leur corpus eZoomBook ;

- une ou deux personnalités historiques et/ou un personnage étranger faisant l'expérience de Nantes devaient être inclus, etc.

\footnotetext{
${ }^{4}$ Sur un total de 32 heures de cours, 24 heures ont été consacrées au projet : cinq sessions de 4 heures ont été consacrées au travail de groupe, 4 heures étant consacrées à la présentation orale des eZoomBooks et des circuits Baludik. Les 4 heures restantes ont été utilisées pour le "défi Baludik». Les ressources générées par les étudiants ont été partagées sur un padlet (https://fr.padlet.com/ vermamayank293/thebaludikchallenge). L'enseignant a pu inclure l'évaluation du projet dans la note finale du semestre : le travail sur eZoomBook ou Baludik terminé comptait pour $25 \%$ de la note finale.
} 
Les groupes de 6 à 8 étudiants étaient composés des équipes suivantes :

- les organisateurs (chargés de l'organisation de la manifestation),

- les «reporters» (chargés du compte rendu de la préparation de la manifestation et de la manifestation elle-même),

- les 6 équipes eZoomBook et 6 équipes de circuit.

Le groupe d'organisateurs a défini un ensemble de règles indiquant notamment quelles équipes joueraient les unes contre les autres et comment elles marqueraient des points.

Nous avons recueilli les commentaires des étudiants sur le projet à des fins de recherche et pédagogiques, notre souhait étant d'améliorer le projet à l'avenir. En ce qui concerne nos recherches, nous voulions mesurer les avantages de l'expérience pour travailler sur la compréhension des genres. Nous voulions également tester l'importance de telles activités en termes d'implication au sein du groupe et d'intégration culturelle.

\section{MÉTHODOLOGIE D’ENQUÉTE}

Les commentaires des étudiants ont été recueillis via les démarches qualitative et quantitative précédemment mentionnées. Le sondage Google comprenait 43 questions. Les questions et réponses sur lesquelles s'appuie notre analyse figurent sur la page de blog du projet (voir notre bibliographie ${ }^{5}$ ). Par ailleurs, nous avons organisé les résultats selon l'articulation suivante :

- compréhension des consignes et partie théorique,

- analyse des contenus (médiation de texte, créativité et progression en langue, etc.) et résultats du sondage Google sur le défi Baludik et activité eZoomBook,

- réticences ou résistances face à l'exercice.

Ces trois points nous permettront également d'aborder les questions concernant la collaboration au sein du groupe, l'autonomie des étudiants, le compétences techniques (création et édition de livres électroniques).

\section{OBSERVATIONS ET RÉSULTATS}

Compréhension des consignes et partie théorique :

Notre approche s'appuyant sur la pratique, la partie théorique du projet s'est limitée aux consignes de la première séance et à un exposé succinct sur les genres

\footnotetext{
${ }^{5}$ Notre blog rapporte l'ensemble de nos expériences pédagogiques : https://ezbresearch.hypotheses. org/students-contribution. L'un des onglets se concentre sur l'expérience eZoomBook et Baludik: https://ezbresearch.hypotheses.org/workshops-with-centrale-students.
} 
littéraires à exploiter lors de la création des histoires. Les étudiants ont compris les informations qui leur ont été communiquées, puis sont rapidement passés à la pratique : c'est en élaborant leur eZoomBook ou circuit que les étudiants ont véritablement intégré les éléments théoriques du projet, notamment les questions autour des codes d'écriture. C'est par la pratique également qu'ils ont démontré leur capacité à établir des ponts entre les deux types de production (eZoomBook et Baludik), de genres radicalement différents.

Analyse des contenus :

L'analyse des productions des étudiants révèle plusieurs points intéressants mis en lumière dans les tableaux sur la page de blog du projet (précédemment citée) :

- Les productions sont systématiquement des genres hybrides, s'appuyant à la fois sur les genres littéraires et les jeux numériques (Baludik et eZoomBook).

- Aucun des genres produits par les étudiants n'appartient à l'un des deux genres vers lequel nous les avions orientés par nos consignes.

- Le dénominateur commun de toutes les productions est l'humour.

En effet, c'est en s'écartant des codes d'écriture énoncés pour leur eZoomBook et Baludik que les étudiants ont créé des effets comiques. Par exemple, l'équipe « Le road trip », a introduit un style d'écriture qui ressemble au script de Bollywood ou, l'équipe «Anne's Ghost», s'est inspiré des films d'horreur. Notre tableau sur la page de blog du projet récapitule les différents genres littéraires et codes dans lesquels les étudiants ont puisé.

La question qui se pose lorsque l'on examine ces productions des étudiants est : à quels genres nouveaux appartiennent-ils ? Réponse : à des genres hybrides, parodiques, originaux, souvent inspirés de formes cinématographiques et qu'il est parfois difficile d'identifier clairement. Par exemple, l'histoire d'amour écrite par l'équipe « Le road trip » constitue un genre inédit que nous avons appelé « Bollywood-àNantes ». Il est, en effet, intéressant de pousser l'exercice jusqu'à la production d'étiquettes pour caractériser les genres que les étudiants semblent avoir produits parfois de manière fortuite.

Pour ce qui est de la dimension multimodale des productions des étudiants, nous observons que l'intégration d'enrichissements est systématique et qu'elle révèle de différentes stratégies : Pour «L'amour éternel », l'équipe s'est attachée à trouver des enrichissements en lien avec la ville de Nantes. Pour « Le road trip », c'est la narration qui tient la première place. Quant au récit «Anne's Ghost», il met en lumière des pages d'histoires inscrites dans des lieux touristiques, tout comme « Le café » qui souhaite pourtant s'inscrire dans un contexte plus moderne.

Les enrichissements des eZoomBooks, sont variés dans la forme et le fond et révèlent la liberté donnée aux étudiants de piocher dans différentes sources ainsi que de créer leurs propres supports (enregistrements sonores et vidéos). 
Résultats du questionnaire :

Les résultats du questionnaire sont disponibles sur le blog relatif au projet. Les questions ${ }^{6}$ se répartissent en deux catégories :

- les premières questions portent sur le profil des élèves, en termes d'habitudes de lecture et de références culturelles et montrent que notre cible n'a pas l'habitude de lire des romans, ni dans leur langue maternelle, ni en anglais (90\% des étudiants lisent moins de 4 romans par an) ;

- les questions $28,29,30,31$ et 33 nous permettent de comprendre comment notre projet est perçu et c'est principalement sur ces questions-là que portera notre analyse.

Les réponses de nos étudiants comprennent les commentaires, que nous avons reproduits sur la page de blog du projet, sans corriger l'anglais, afin de préserver l'authenticité de la matière.

Quelques mots sur le profil des étudiants :

Tous les étudiants sont issus des programmes de master en ingénierie de Centrale Nantes. Ils viennent d'un grand nombre de pays : $90 \%$ des étudiants viennent d'autres pays que l'Europe et $80 \%$, d'Inde et du Pakistan. La plupart ne sont pas enclins à la lecture : ils lisent moins de 4 livres par an. Leurs productions créatives précédemment analysées mettent en lumière leur filtre cognitif (par exemple, l'histoire d'amour écrite par un groupe d'étudiants indiens constitue un genre nouveau inspiré de leur culture d'origine, que nous avons appelé «Bollywood-à-Nantes»). Beaucoup de nos questions se posent concernant leur filtre cognitif - par exemple : «Comment l'histoire coloniale des pays des étudiants affecte-t-elle leur perception de Nantes? », cependant il serait difficile pour les étudiants d'y répondre de manière objective. C'est pourquoi nous nous sommes contentés de les interroger sur leur travail au cours du projet et sur leurs motivations.

Perception du projet :

Les questions visant la perception du projet par les étudiants ont été formulées en fonction de notre cible d'étudiants étrangers. Nous avons notamment travaillé la langue du questionnaire pour le rendre accessible aux étudiants. Par exemple, l'expression «Wow moment», que nous pourrions traduire par «moment d'émerveillement » était celle que les étudiants avaient eux-mêmes utilisée en classe et nous l'avons reprise pour l'inclure dans notre questionnaire.

Les principaux résultats quantitatifs se résument en trois points :

- Les étudiants perçoivent les outils proposés (eZoomBook et Baludik) comme des outils qui permettent de mieux découvrir des lieux touristiques ${ }^{7}$.

\footnotetext{
${ }^{6}$ Le questionnaire est disponible sur la page suivante: https://ezbresearch.hypotheses.org /workshops-with-centrale-students.
} 
- Le lien entre les textes et les lieux est fortement souligné ${ }^{8}$.

- Sur le plan de la réception, l'invitation à « bouger » et « se rendre sur le site du texte » stimule l'activité de lecture.

Les chiffres précis sont donnés sur le blog relatif au projet.

Implication, réticences ou modes de résistance développés au sein du groupe :

Nous avons observé combien les étudiants étaient impliqués dans le projet, participant aux échanges, effectuant des recherches et demeurant concentrés sur le travail à faire pendant les séances consacrées à cette activité. Ainsi, le projet tel qu'il était proposé a recueilli l'adhésion des étudiants et les a amenés à entreprendre une activité d'écriture dont ils ont bien cerné les objectifs pédagogiques (en termes de pratique de la langue et d'activité de recherche culturelle).

Néanmoins, les échanges ont rapidement fait apparaitre d'autres motivations au sein du groupe, notamment le fait de créer un récit ou bien un parcours conçu non pas pour instruire mais pour amuser leurs camarades de classe. Est apparu alors, au fur et à mesure du travail, un mode de résistance face au contexte scolaire : le projet a été, en quelque sorte, détourné de sa fonction pour devenir un outil d'expression d'individualité.

Face à ces résistances constatées, nous nous sommes posés la question de ses «lignes de fuite ${ }^{9}$ du projet, pour reprendre l'expression de Deleuze et Gattari (1972) en référence à la théorie de Foucault (1969) : il nous a semblé que ces lignes de fuite offraient autant d'évolutions possibles pour la suite de l'expérience car le projet nous fournissait, de manière inattendue, une matière qui venait renforcer nos objectifs de créativité et d'innovation.

En les regardant travailler, nous avons remarqué d'emblée que les étudiants étaient à la recherche du ludique : ce qui leur plaisait c'était de créer quelque chose qui allait surprendre leurs camarades et les faire rire. Ainsi, ayant entre les mains un outil d'édition multimodal à potentiel à la fois instructif et ludique, ils se sont focalisés sur les éléments ludiques et ils ont atteint les objectifs des enseignants par un chemin inattendu : en passant par une légère transgression des consignes, et en

\footnotetext{
${ }^{7}$ Par exemple, notons les réponses à la question : « Est-ce que ces outils contribuent à susciter des moments d'émerveillement au cours de la visite, la majorité des étudiants répondent affirmativement : 2 «non » et 2 « pas vraiment », 3 « peut-être » 13 « sans réponse » et 41 « oui » (with or without extra comments).

${ }^{8}$ La majorité des étudiants répondent affirmativement à la question : « La lecture d'un texte portant sur un lieu permet-elle d'aiguiser votre perception du lieu ?»: 3 «non» et 1 «pas vraiment», 2 «peutêtre 12 « sans réponse » et 45 « oui ».

${ }^{9}$ Les lignes de fuite peuvent se définir comme les lignes qui établissent une construction en perspective (comme pour les lignes de fuite d'une peinture classique) et qui constituent une sorte de mouvement de libération par rapport à la structure établie. En ce qui concerne notre projet, les « lignes de fuite » se manifestent par tout ce que les étudiants apportent à leur travail et qui se situe en-dehors des consignes. Nous souhaitons prendre en compte ces éléments pour les éditions futures du projet.
} 
élaborant des contenus susceptibles de plaire à leur cible de lecteurs. On peut s'interroger sur les motivations de ce comportement observés systématiquement chez tous les groupes de la classe : plaisir de la transgression ou approbation de leurs pairs? Tout autant que le plaisir du jeu et les théories de " gamification », la théorie des faces nous semble ici particulièrement pertinente : afin de " conserver la face », les étudiants souhaitaient éviter l'écueil d'une production littéraire médiocre et proposer à la place un eZoomBook ou un circuit amusant (Goffman, 1974). C'est ce que semblent indiquer leurs commentaires au moment du feedback qualitatif (du type "We wanted to be entertaining », "We wanted the group to enjoy our story/circuit »). Le désir de remporter l'adhésion de leur public le jour du "défi Baludik» fût, par ailleurs, encouragé par l'équipe des «organisateurs » qui, eux aussi, souhaitaient "sauver la face» en offrant à leurs pairs un événement distrayant.

Ainsi, si nous attendions, à l'issue de cet exercice, une conformité à un genre donné - soit le genre narratif à composante touristique (pour les équipes eZoomBook), soit le genre du questionnaire touristique (pour les équipes Baludik), les étudiants se sont plutôt livrés à un jeu de parodie et d'hybridation des genres. Les enseignants ont tiré parti de ce résultat inattendu, tant ils étaient convaincus que l'on apprend autant sur les genres du discours en jouant avec ces genres qu'en cherchant à s'y conformer. Les enseignants étaient, par ailleurs, également convaincus qu'il était impossible de réussir dans le genre fictionnel sans être un auteur de talent (ce qui ne correspondait pas au profil de nos étudiants ingénieurs). Ainsi, dans les pistes d'amélioration du projet, nous avons souhaité inclure une séance d'échange qualitatif supplémentaire pour exploiter les résultats inattendus. La transgression des étudiants, favorablement accueillie par les enseignants, pourrait servir de prétexte à un apport théorique supplémentaire et c'est ce que nous nous proposons d'explorer dans nos pistes d'amélioration du projet.

\section{PISTES D'AMÉLIORATION DU PROJET}

Les améliorations que nous souhaitons mettre en place à l'issue du projet se situent à la fois sur le plan pratique et sur le plan de la réflexion proposée aux étudiants.

Améliorations pratiques :

Une amélioration des outils techniques s'impose. Pour l'éditeur de circuits, celle-ci est en cours (voir les derniers développements sur le site internet Baludik). Pour l'outil eZoomBook, les prochains développements visent une amélioration de l'interface utilisateur et l'introduction de nouvelles fonctionnalités (comme la possibilité d'établir des hyperliens entre les différentes couches du texte, de manière 
automatique). Les évolutions du projet de recherche eZoomBook seront valorisées sur le site de notre bibliographie.

Au-delà de ces deux développements techniques indispensables au projet, nous avons pensé à la création d'outils supplémentaires pour faciliter le travail linguistique : pour une prochaine édition, nous pensons mettre à la disposition des étudiants quelques supports pédagogiques dans lesquels ils pourront piocher en toute autonomie : par exemple, un lexique spécifique, des listes de phrases type pour la rédaction d'énigmes, etc.

Réflexion sur les genres de discours :

Le retour d'expérience est l'occasion d'offrir aux étudiants une réflexion supplémentaire, par deux biais essentiels :

- le réfléchissement du comportement des différents groupes lorsqu'ils effectuent la tâche demandée et qu'ils proposent leur travail au groupe (il s'agit en quelque sorte, de tendre un miroir aux participants),

- l'introduction d'un apport théorique supplémentaire sur la théorie des genres littéraires (cet apport théorique s'appuie directement sur l'expérience).

Le miroir tendu aux étudiants leur permet de constater qu'ils se comportent comme des locuteurs qui, face à la difficulté d'un genre donné (l'écriture de fiction), contournent l'obstacle en jouant sur la parodie et les effets comiques. Ainsi, ils sont amenés à comprendre que la maîtrise d'un genre de discours passe par l'imitation. Comme le soulignait le linguiste russe Bakhtine : « Nous apprenons à mouler notre parole dans les formes du genre et, entendant la parole d'autrui, nous savons d'emblée, au tout premier mot, en pressentir le genre, en deviner le volume, la structure compositionnelle donnée, en prévoir la fin, autrement dit, dès le début nous sommes sensibles au tout discursif [...] Si les genres de discours n'existaient pas et si nous n'en n'avions pas la maîtrise, et qu'il nous faille les créer pour la première fois dans le processus de la parole, et qu'il nous faille construire chacun de nos énoncés, l'échange verbal serait impossible » (Bakhtine, 1984, p. 285).

Nous l'avons vu, les genres d'écriture exigés par nos deux outils - qu'ils soient les genres du roman d'aventure, des films de Bollywood, des jeux numériques, de la brochure touristique ou bien d'autres encore - sont très différents (bien qu'ils offrent tous deux la possibilité au lecteur de se balader entre les hyperliens du texte) : le concepteur Baludik travaille sur un seul niveau de texte très court, tandis que la tâche est plus complexe pour le concepteur du eZoomBook : il lui faut apprendre à écrire de manière à accrocher l'attention flottante des lecteurs, de manière à le séduire et à le garder captif du récit.

Si les étudiants ne se plient pas entièrement aux consignes, ils savent, malgré tout, percevoir l'essentiel : l'exercice qui leur est proposé est une exploration des genres du discours. En effet, le projet leur fait toucher du doigt que ces genres sont des contrats de nature foncièrement coopérative et réglés par des normes. Les 
étudiants perçoivent également que la nature de la coopération peut être transgressive : eux-mêmes prennent plaisir à aller contre l'horizon d'attente du lecteur et ils vont jusqu'à à briser le premier contrat du genre du discours imposé au profit d'un deuxième genre qui est celui de leur choix et qui correspond à une catégorie hybride.

Réflexions complémentaires :

L'apport théorique supplémentaire peut comprendre des questions concernant le medium, la forme (orale ou écrite), la dynamique de l'échange verbal, les composantes de la scène d'énonciation, etc. Ainsi, les pistes de réflexions complémentaires comprennent les quelques points suivants.

Lien entre médium et discours :

C'est surtout avec l'avènement des médias audiovisuels et le développement de l'informatique que nous prenons conscience de ce rôle crucial du médium. La nature des textes et leurs modes de consommation s'en trouve bouleversés. Les étudiants deviennent davantage conscients de ce fait lorsqu'ils sont en situation de créer des contenus pour les différents médias. Ils peuvent s'observer en tant que «natifs digitaux » (Palfrey, Gasser, 2008), s'interroger sur leur mode d'interaction avec les outils que nous leur proposons et sur ceux qu'ils ont l'habitude d'utiliser de manière spontanée. Les rôles et les postures dans le processus d'apprentissage suscitent alors bien des interrogations de la part des enseignants comme des apprenants (Laurillard, 2009 ; Davison, 2011). Cette réflexion débouche, dans le cadre de notre projet, sur un débat autour des dangers soulignés par Turkle, dans le titre même de son ouvrage: Alone together (2011). Ce livre met l'accent sur le lien entre une forte consommation des outils digitaux et une régression en termes de compétences sociales. Nos étudiants soulignent alors l'importance de la composante « histoire » dans le projet Baludik et eZoombook pour ne pas restreindre l'exercice à un jeu digital solitaire et pour, au contraire, renforcer les interactions extra-digitales entre pairs.

Opposition entre oral et écrit :

Le projet les amène également à faire la distinction entre l'oral et l'écrit : les circuits Baludik, lorsqu'ils sont proposés à un groupe, tablent sur l'interaction des participants. Si la distinction entre l'oral et l'écrit est la catégorie médiologique la plus ancienne et la plus solidement ancrée dans la culture, cette distinction n'est pas si simple qu'on pourrait le penser: les contenus enrichis des circuits invitent à dépasser l'opposition écrit oral, car l'écrit de l'application Baludik devient oral lorsque les questions inscrites à chaque étape sont lues à haute voix. Elles sont donc conçues autant pour l'oral que pour l'écrit puisqu'il s'agit, en les écrivant, de se projeter dans une situation orale. De plus la question de la stabilité de l'énoncé écrit 
et remise en question du fait de la dynamique de l'échange verbal autour de la résolution d'énigmes, la découverte d'indices etc. Enfin, la distinction entre oral et écrit fait également intervenir une distinction entre énoncé dépendant et énoncé indépendant de l'environnement non verbal. L'énoncé Baludik est, par nature, dépendant puisqu'il s'agit de le consommer in situ, en suivant un circuit. L'eZoomBook, quant à lui, est par nature indépendant puisqu'il s'agit d'une œuvre de fiction que l'on peut lire n'importe où. Relier les énoncés Baludik et eZoomBook relève d'un exercice artificiel qui présente un défi conceptuel que les étudiants sont libres de gérer comme ils l'entendent.

Scène englobante et scène générique :

Selon la définition de Maingueneau, ces deux scènes définissent conjointement ce qu'on pourrait appeler le cadre scénique du texte et chacune des deux pose question : comme l'énonce Maingueneau (2000, p. 71) «la scénographie n’est pas simplement un cadre, un décor, comme si le discours survenait à l'intérieur d'un espace déjà construit et indépendant de ce discours, mais l'énonciation en se développant s'efforce de mettre progressivement en place son propre dispositif de parole ». Ainsi la scénographie implique un processus en boucle paradoxale que l'on voit à l'œuvre lors du déroulement des circuits Baludik. Dès son émergence, la parole suppose une certaine situation d'énonciation, laquelle, en fait, se valide progressivement à travers l'énonciation elle-même. « La scénographie est ainsi à la fois ce dont vient le discours [la scène englobante] et ce qu'engendre ce discours [scène générique] » (Maingueneau, 2000, p. 71). Le propos de Maingueneau est donc illustré par la scénographie Baludik qui est en effet «à la fois ce dont vient le discours et ce qu'engendre ce discours; elle légitime un énoncé qui, en retour, doit la légitimer, doit établir que cette scénographie d'où vient la parole est précisément la scénographie requise pour énoncer comme il convient, selon le cas, la politique, la philosophie, la science, ou pour promouvoir telle marchandise » (Maingueneau, 2000, p. 71).

Si l'on ne peut pas préjuger à l'avance des connaissances et repères qui vont être mobilisés lors d'un parcours Baludik, nous avons constaté, en discutant avec les étudiants de Master deux de manière informelle sur les questions de «mise en scène » de leur travail, qu'ils avaient conscience de définir leur propre scénographie en déroulant leur parcours. Ils ont, par ailleurs, souligné une grande diversité de scénographies permettant à chaque équipe des concepteurs du parcours de mettre en situation de narration et de construire la figure du « lecteur idéal » (Iser, 1997 ; Manguel, 2003, 2004) ${ }^{10}$. Cette notion couplée à celle d'horizon d'attente mériterait également d'être explorée avec les étudiants, dans le prolongement de la discussion.

\footnotetext{
${ }^{10} \mathrm{La}$ critique de la réception propose plusieurs termes proches pour différents types de lecteurs : lecteur idéal (Iser, 1997 ; Manguel, 2003, 2004), lecteur implicite (Eco 1985, 1996), lecteur virtuel
} 
Un retour critique sur l'activité que nous proposons pour la suite du projet comprend ainsi une réflexion sur les composantes scénographiques et la figure du lecteur idéal.

\section{CONCLUSION}

Entrepris dans une démarche R-A, notre projet offre quelques éléments inattendus au niveau des productions des étudiants et de l'analyse des données, et ceci n'est pas sans conséquence sur nos préconisations pour l'amélioration du projet. Notre attention se porte donc non seulement sur les questions de motivation et de cohésion de groupe (mesurées par le questionnaire), mais également sur l'exploration des lignes de fuite du dispositif : en effet, il nous semble que le projet présente des richesses inattendues du fait même de la transgression des étudiants à nos consignes. En analysant leur posture, leurs productions en termes d'eZoomBooks et de circuits, ainsi que leur retour sur l'expérience, nous constatons qu'ils se sont donnés toute liberté d'exploration des possibilités de la langue et des genres (le jeu avec les genres était d'ailleurs encouragé par le simple fait que les équipes travaillant en binômes étaient dotées d'outils distincts, visant des productions langagières différentes).

L'innovation des étudiants ayant été accueilli favorablement par les enseignants, notre équipe est en mesure de proposer des pistes sur la manière dont on peut tirer parti de la transgression des étudiants.

Dans ce contexte spécifique (des profils hétérogènes au sein de la classe, peu d'heures, peu d'importance accordée à la matière), il est difficile de pousser plus loin le perfectionnement linguistique et il s'agit plutôt de capitaliser sur le désir des étudiants de gagner l'approbation de leurs pairs et de produire des contenus ludiques, selon la théorie des faces (Goffman, 1974). En effet, au-delà du genre imposé, il importe aux étudiants de provoquer le rire et la surprise. De plus, l'innocence des étudiants face aux médias nouveaux les conduits à inventer de nouvelles règles, renforçant la composante créative de leurs productions.

Selon la préconisation de Lech et Harris dans «Language Learning in the Virtual Wild» (2018), il s'agit d'encourager la diversification des environnements d'apprentissage, et de commencer par une meilleure exploitation des ressources en ligne.

$\mathrm{Au}$ risque de diminuer les mérites des enseignants et des chercheurs qui sont pourtant toujours tendus vers l'amélioration de leur dispositifs pédagogiques, il nous

(Genette, 1991), archilecteur (Riffaterre, 1979). Selon Manguel, «[1]e lecteur idéal ne suit pas une histoire : il y participe» $(2004$, p. 36$)$, et « [u]n livre devient un autre livre chaque fois que nous le lisons » (2003, p. 22). 
semble opportun de citer Rousseau : «Notre manie enseignante et pédantesque est toujours d'apprendre aux enfants ce qu'ils apprendraient beaucoup mieux d'euxmêmes, et d'oublier ce que nous aurions pu seuls leur enseigner. Y a-t-il rien de plus sot que la peine qu'on prend pour leur apprendre à marcher, comme si l'on en avait vu quelqu'un qui, par la négligence de sa nourrice, ne sût pas marcher étant grand ? Combien voit-on de gens au contraire marcher mal toute leur vie, parce qu'on leur a mal appris à marcher !» (1782, p. 426). Ainsi, il nous paraît judicieux, pour conclure au sujet de notre projet, de souligner l'importance de l'aménagement d'espaces de liberté d'exploration et d'expression. Ne faut-il pas, de plus, penser nos prochaines entreprises eZoomBook/Baludik en nous fixant des objectifs délibérément larges afin d'encourager l'initiative de chaque apprenant ? 


\section{BIBLIOGRAPHIE}

Bakhtine, M.M. (1984). Esthétique de la création verbale. Paris : Gallimard.

Bellay, C., Marco, C., \& Evain, C. (2017). eZoomBook Methodology and Template. A Case Study of Collective Authorship in the Classroom. Studies in English Language Teaching, 5, https://doi.org/10.22158/selt.v5n2p231.

Bon, F. (s. d.). Le tiers livre, web \& littérature. Google n'annule pas Borges, il l'exacerbe. Consulté 18 novembre 2018, à l'adresse http://www.tierslivre.net/spip/spip.php?article2744.

Davidson, C.N. (2011). Now you see it: how the brain science of attention will transform the way we live, work, and learn. New York : Viking.

Deleuze, G., \& Guattari, F. (1980). Mille plateaux : capitalisme et schizophrénie. Paris : Les Editions de Minuit.

Eco, U. (1985). Lector in fabula : le rôle du lecteur ou La coopération interprétative dans les textes narratifs (trad. M. Bouzaher). Paris : Librairie Générale Française.

Eco, U. (1992). Les Limites de l'interprétation. Paris : Grasset.

Evain, C., \& De Marco, C. (2015). Rethinking the promotion of reading. The BRIO eZoomBook workshop. 9th International Technology, Education and Development Conference, INTED 2015 Proceedings (pp. 7926-7933). Valencia : IATED.

Evain, C., De Marco, C., \& Carolan, S. (2013). Le nouveau dispositif « eZoomBook » : perspectives pédagogiques. Distances et Médiations des Savoirs, 3, 1-17.

Evain, C., De Marco, C., \& Carolan, S. (2014). Reading with a difference. eZoomBook and MyGame-4. Publishing Research Quarterly, 30 (1), 50-62.

Flahault, F. (1978). La parole intermédiaire. Préf. de Roland Barthes. Paris : Ed. du Seuil.

Foucault, M. (1969). Archeologie Du Savoir. Gallimard.

Goffman, E. (1974). Les rites d'interaction (trad. A. Kihm). Paris : Ed. de Minuit.

Iser, W. (1997). L'acte de lecture: théorie de l'effet esthétique. Sprimont : Mardaga.

Iwaszko, I., Lojkine, P., Evain, C., De Marco, C. (2018). Le livre numérique enrichi eZoomBook et ses applications dans le champ scolaire. Revue de recherches en littératie médiatique multimodale, 8 , https://itmedmod.ca/le-livre-numerique-enrichi-ezoombook-et-ses-applicationsdans-le-champ-scolaire.

Laurillard, D., University of London, \& Institute of Education (2008). Digital technologies and their role in achieving our ambitions for education. London : University of London, Institute of Education.

Lech et Harris dans « Language Learning in the Virtual Wild. To appear as: Lech, I.B., \& Harris, L.N. (2019). Language learning in the virtual wild. In M.L. Carrió-Pastor (Ed.), Teaching language and teaching literature in virtual environments (pp. 39-54). Singapore : Springer. DOI : 10.1007/978-981-13-1358-5.

Louichon, B. (2015). Le patrimoine littéraire : un enjeu de formation. Tréma, 43, 22-31, https://doi.org/10.4000/trema.3285.

Maingueneau, D. (2000). Analyser les textes de communication. Paris : Nathan.

Manguel, A. (2003). Dans la forêt du miroir : essais sur les mots et sur le monde. Arles : Actes Sud.

Manguel, A., Le Bœuf, C., \& Manguel, A. (2004). Journal d'un lecteur. Arles : Actes Sud.

Marco, C., \& Evain, C. (2015). Rediscovering and promoting serious reading. How the eZoomBook platform can help, vol. 8.

Narcy-Combes, J.-P. (1998). La problématique action research/recherche-action et le travail coopératif. GERAS Groupe d'Etude de Recherche en Anglais de Spécialité, 229-238. 
Narcy-Combes, J.-P. (2001). La recherche-action en didactique des langues : apprentissage, compagnonnage ou évolution libre? Recherche et pratiques pédagogiques en langues de spécialité. Cahiers de l'Apliut, vol. XXI, no. 2, 40-52, https://doi.org/10.4000/apliut.4639.

Palfrey, J.G., \& Gasser, U. (2008). Born digital: understanding the first generation of digital natives. New York : Basic Books.

Riffaterre, M. (1979). La production du texte, vol. 1-1. Paris : Éditions du Seuil.

Rousseau, J.-J. (1782). Collection complete des oeuvre de J.-J. Rousseau. Geneve : Samuel Fauche.

Turkle, S. (2011). Alone together: why we expect more from technology and less from each other. New York : Basic Books. 